Rev. Inst. Flor. v. 29 n. 2 p. $253-261$ dez. 2017

http://dx.doi.org/10.24278/2178-5031.201729209

ISSN impresso 0103-2674/on-line 2178-5031

\title{
VARIABILIDADE GENÉTICA EM CLONES DE Eucalyptus spp. PARA GRAJAÚ, ESTADO DO MARANHÃO ${ }^{1}$
}

\section{GENETIC VARIABILITY IN CLONES OF Eucalyptus spp. FOR GRAJAÚ, STATE OF MARANHÃO}

\author{
Evandro Vagner TAMBARUSSI ${ }^{2,3,4}$; Fernanda Bortolanza PEREIRA ${ }^{3}$; \\ Vinícius André AZEVEDO²; Fabiana Schmidt Bandeira PERES²; \\ Andrea Nogueira DIAS ${ }^{2}$; Rodrigo de Andrade FURLAN ${ }^{3}$
}

\begin{abstract}
RESUMO - Esta pesquisa teve por objetivo a seleção de clones de eucaliptos em teste em Grajaú-MA, Brasil, visando ao aumento na produtividade. Os caracteres avaliados foram diâmetro à altura do peito - DAP $(\mathrm{cm})$ e altura - ALT $(\mathrm{m})$. Para isto, um total de 130 clones, distribuídos em 20 blocos, com uma planta/parcela, foram mensurados aos quatro e cinco anos. Os procedimentos estatísticos foram o método do modelo misto para obter o melhor preditor linear não viesado (BLUP) e a máxima verossimilhança restrita (REML) para estimar os componentes de variância. Para clones e parcelas, os testes de razão de verossimilhança (LRT) foram significativos a $1 \%$ de probabilidade. A variabilidade genética foi alta e variou de $11,42 \%$ a $14,70 \%$ para DAP e $9,15 \%$ a $10,22 \%$ para ALT. Os coeficientes de variação relativa $(C V)$ foram maiores que um para ambos os caracteres, mostrando que a seleção pode resultar em grandes e rápidos ganhos genéticos. No geral, os caracteres foram altamente acurados. As correlações genéticas $\left(\hat{r}_{g}\right)$ foram positivas, indicando a possibilidade de seleção indireta. Os resultados indicaram que o DAP deve ser usado preferencialmente em relação à ALT, e permitem concluir que os ganhos genéticos após a seleção são muito promissores. Os clones selecionados podem ser indicados para testes clonais piloto e/ou fase II em locais com condições ambientais similares às do local de teste.
\end{abstract}

Palavras-chave: melhoramento florestal; parâmetros genéticos; herdabilidades; correlação.

\begin{abstract}
This research aimed to select clones of eucalypts under testing in Grajaú, MA, Brazil, in order to increase the wood productivity. The traits considered were diameter at breast height - DAP $(\mathrm{cm})$ and height - ALT $(\mathrm{m})$. For this purpose, a total of 130 clones, distributed across 20 blocks with one plant/plot, were assessed at the ages four and five years. The statistical procedures were the mixed model method to obtain the best linear unbiased predictors (BLUP) and the restricted maximum likelihood (REML) to estimate the components of variance. For clones and plots, the likelihood ratio tests (LRT) were significant at $1 \%$ of probability. The genetic variability was high and ranged from $11.42 \%$ to $14.70 \%$ for DAP and $9.15 \%$ to $10.22 \%$ for ALT.
\end{abstract}

\footnotetext{
${ }^{1}$ Recebido para análise em 13.07.17. Aceito para publicação em 20.12.17.

${ }^{2}$ Universidade Estadual do Centro-Oeste, BR 153, Km 7, Bairro Riozinho, 84500-000, Irati, PR, Brasil.

3Programa de Pós-Graduação em Ciência Florestal (FCA/UNESP) - Rua José Barbosa de Barros, 1780, Portaria II: Rodovia Alcides Soares, Km 3, 18610-307,

Botucatu, SP, Brasil.

${ }^{4}$ Autor correspondente: Evandro Vagner Tambarussi - tambarussi@gmail.com
} 
The coefficient of variation ratio $\left(C V_{r}\right)$ was greater than one for both traits, showing that selection can result in large and rapid genetic gains. In general, the traits were highly accurate. The genetic correlations $\left(\hat{r}_{g}\right)$ were positive, indicating the possibility for indirect selection. The results indicated that DAP must be used preferentially in relation to ALT, and allow us to conclude that the genetic gains after selection are very promising. The selected clones can be indicated to pilot clonal tests and/or phase II to places with environmental conditions similar to the field trial.

Keywords: forest breeding; genetic parameters; heritability; correlation.

\section{INTRODUÇÃO}

No Brasil, os plantios florestais começaram há mais de um século quando o pioneiro Engenheiro Agrônomo Edmundo Navarro de Andrade trouxe, em 1903, as primeiras mudas de eucalipto para os plantios que produziriam madeira para os dormentes das estradas de ferro (Martini, 2008). Com uma área de 7,8 milhões de hectares com árvores plantadas, é responsável por $91 \%$ de toda a madeira produzida para fins industriais no país (Indústria Brasileira de Árvores - IBÁ, 2016). Nos últimos quatro anos, o crescimento da área com árvores plantadas foi de $6 \%$, em comparação com os 7,39 milhões de hectares de 2012 (IBÁ, 2014). Os plantios de eucalipto ocupam 5,6 milhões de hectares da área total com florestas plantadas do País e estão localizados principalmente em Minas Gerais (24\%), São Paulo (17\%) e no Mato Grosso do Sul (15\%) (IBÁ, 2016).

Com o passar do tempo, o Brasil fomentou e desenvolveu tecnologias no setor florestal que o tornou mais competitivo em relação a outros países na produção de florestas de eucalipto, bem como o tornou um do mais relevantes no cenário global (Associação Brasileira de Produtores de Florestas Plantadas - ABRAF). O sucesso no cultivo de eucalipto deve-se também ao melhoramento florestal, associado a técnicas silviculturais apropriadas (Pinto Júnior et al., 2005; Xavier e Silva, 2010), bem como a ferramentas biotecnológicas (Andrade et al., 2018).

Para alcançar de forma homogênea maior produtividade das florestas plantadas de eucalipto, houve a necessidade da implantação de clones, satisfazendo, assim, a crescente demanda industrial para o setor (Berger et al., 2002).
Particularmente, o Brasil, a seleção de clones híbridos que correspondam às necessidades do ponto de vista silvicultural e tecnológico recebe grande ênfase nos programas de melhoramento genético (Nunes et al., 2002; Tambarussi et al., 2017). A produção de híbridos interespecíficos em Eucalyptus spp., com a utilização de espécies que se adaptam a regimes de baixa precipitação pluviométrica, é prática comum no melhoramento de espécies deste gênero (Verryn, 2000).

A relativa carência de informações sobre o desenvolvimento de florestas plantadas nos estados do Nordeste brasileiro comparativamente a outras regiões mais tradicionais de cultivo de eucaliptos, requer estudos mais amplos com relação às espécies e procedências de rápido crescimento e possibilidade de adaptação, para que se torne viável a atividade comercial de reflorestamento (Miranda, 2012).

As empresas que estão plantando eucaliptos clonais no estado do Maranhão se valem basicamente de híbridos que incluam a espécie Eucalyptus urophylla S.T. Blake, devido à sua rusticidade e resistência a doenças tropicais. Entre esses híbridos se destacam E. urophylla $\mathrm{x}$ Eucalyptus grandis W. Hill ex Maiden e (Eucalyptus camaldulensis Dehnh. x E. grandis) x E. urophylla (Miranda, 2012).

Desse modo, este trabalho teve por objetivo selecionar clones com melhor taxa de crescimento em um teste clonal de Eucalyptus spp., aos quatro e cinco anos de idade, utilizando metodologia de modelos mistos para obtenção do melhor preditor linear não visado (BLUP) e o procedimento de máxima verossimilhança restrita (REML) para obtenção dos componentes de variância. 


\section{MATERIAL E MÉTODOS}

\subsection{Ensaio de Campo e Material Genético}

Os dados utilizados na presente pesquisa são provenientes de um teste clonal de Eucalyptus spp. sob responsabilidade do Grupo Ferroeste. O experimento está instalado

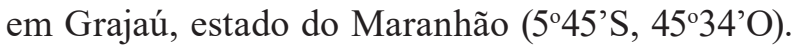
O local está sob domínio de clima tropical, com estação seca, do tipo Aw, segundo a classificação de Köppen-Geiger, apresentando verão chuvoso e inverno seco, com precipitação do mês mais seco inferior a $60 \mathrm{~mm}$ e precipitação média anual em torno de $1.200 \mathrm{~mm}$, concentrados principalmente no primeiro trimestre do ano (Lima et al., 1999). A temperatura média do mês mais frio é superior a $18{ }^{\circ} \mathrm{C}$ e a média anual está em torno de $26{ }^{\circ} \mathrm{C}$. Os caracteres avaliados aos quatro e cinco anos de idade foram o diâmetro a altura do peito - DAP (em $\mathrm{cm})$ e altura - ALT (em m) para 130 clones (tratamentos), distribuídos em 20 blocos com uma planta/parcela. Os materiais genéticos são clones selecionados oriundos do cruzamento de E. grandis $\mathrm{x}$ E. urophylla.

\subsection{Estimativa dos Parâmetros Genéticos}

Os valores genotípicos de cada caractere analisado foram estimados utilizando o software Selegen (Resende, 2007a), a partir do procedimento da Máxima Verossimilhança Restrita (REML) empregando o modelo linear misto para avaliação de clones não aparentados:

$$
\mathbf{Y}=\mathbf{X b}+\mathbf{Z g}+\mathbf{W} \mathbf{p}+\mathbf{e}
$$

em que: $\mathbf{Y}=$ vetor de observações fenotípicas para um caráter; $\mathbf{X}=$ matriz de incidência para os efeitos fixos; $\mathbf{b}=$ vetor dos efeitos fixos (média geral e efeitos de bloco); $\mathbf{Z}=$ matriz de incidência para os efeitos genotípicos; $\mathbf{g}=$ vetor dos efeitos genotípicos; $\mathbf{W}=$ matriz de incidência dos efeitos da parcela; $\mathbf{p}=$ vetor dos efeitos da parcela e $\mathbf{e}=$ vetor dos erros ou resíduos (aleatórios).
As equações do modelo misto para estimar os efeitos fixos e predizer os efeitos aleatórios são dadas por:

$$
\left[\begin{array}{l}
\hat{\beta} \\
\hat{g} \\
\hat{p}
\end{array}\right]=\left[\begin{array}{ccc}
X^{\prime} X & X^{\prime} Z & X^{\prime} W \\
Z^{\prime} X & Z^{\prime} Z+G^{-1} \sigma_{a d}^{2} & Z^{\prime} W \\
W^{\prime} X & W^{\prime} Z & W^{\prime} W+I \lambda_{5}
\end{array}\right]^{-1}\left[\begin{array}{c}
X^{\prime} y \\
Z^{\prime} y \\
W^{\prime} y
\end{array}\right],
$$

e as predições dos valores genotípicos de cada clone são obtidas por:

$\hat{g}_{i}=h_{c}^{2}\left(\bar{Y}_{i \ldots}-\bar{Y}_{\ldots}\right)$

em que:

$h_{c}^{2}=\left(\sigma_{a}^{2}+\sigma_{d}^{2}\right) /\left[\left(\sigma_{a}^{2}+\sigma_{d}^{2}\right)+\sigma_{e}^{2} / b_{i}+\sigma_{a d}^{2} / \sum n_{i}\right]$; $\sigma_{a}^{2}=$ variância genética aditiva; $\sigma_{d}^{2}=$ variância genética de dominância; $\sigma_{e}^{2}=$ variância genética entre parcelas; $b_{i}=$ efeito do bloco; $\sigma_{a d}^{2}=$ variância ambiental dentro de parcela.

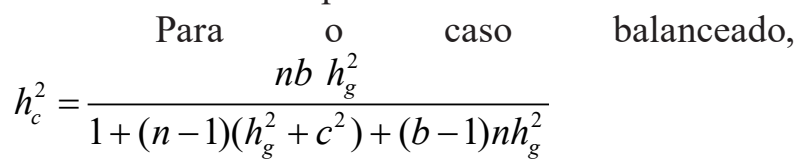
em que $\mathrm{n}$ e $\mathrm{b}=$ número de plantas/parcela e de blocos associados à avaliação do material, respectivamente.

O método REML foi utilizado por meio de algoritmos EM (esperança e maximização), que realizam estimativas dos efeitos ajustados dos vetores calculados por meio de resoluções de matrizes. Assim, foram estimados os seguintes parâmetros:

a) Variância ambiental entre parcelas $\left(\hat{V}_{c}\right)$ : $\hat{V}_{c}=\left[\hat{c}^{\prime} \hat{c}+\hat{V}_{e} \operatorname{tr} C^{33}\right] / s_{1}$;

b) Variância residual (ambiental + não aditiva) $\left(\hat{V}_{e}\right)$ : $\hat{V}_{e}=\left[y^{\prime} y-\hat{r}^{\prime} X^{\prime} y-\hat{a}^{\prime} Z^{\prime} y-\hat{c}^{\prime} W^{\prime} y\right] /[N-r(x)]$;

c) Herdabilidade no sentido amplo $\left(h_{g}^{2}\right): h_{g}^{2}=\frac{\hat{V}_{g}}{\hat{V}_{f}}$;

d) Coeficiente dos efeitos ambientais entre parcelas: $c_{\text {parc }}^{2}=\frac{\hat{V}_{p}}{\hat{V}_{g}+\hat{V}_{p}+\hat{V}_{e}} ;$

e) Herdabilidade média de clones: $h_{m}^{2}=\frac{V_{g}}{V_{g}+\frac{V_{c}}{b}+\frac{V_{e}}{n b}}$;

f) Coeficiente de variação experimental: $C V_{\exp }(\%)=\frac{\sqrt{\frac{V_{e}}{n}+V_{c}}}{m} .100$; 
g) Coeficiente de variação genética: $C V_{g}(\%)=\frac{\sqrt{V_{g}}}{m} \cdot 100$;

h) Relação entre o coeficiente de variação genético pelo ambiental: $C V_{r}=\frac{C V_{g}(\%)}{C V_{\exp }(\%)}$;

i) Variância genética: $\hat{V}_{g}=\hat{V}_{a}+\hat{V}_{d}+\hat{V}_{I}$, em que $\hat{V}_{a}$ variância genética aditiva; $\hat{V}_{d}$ variância genética dominante e $\hat{V}_{I}=$ variância genética epistática.

\subsection{Correlação Genética e Fenotípica}

A correlação genética, $r_{g}$ ou rggd, entre dois caracteres ou um único caráter em idades distintas foi estimada por $\hat{r}_{g}=\frac{C \hat{O} V}{\sqrt{\hat{\sigma}_{(x)}^{2} \cdot \hat{\sigma}_{(y)}^{2}}}$, em que a $C \hat{O} V_{a(x, y)}$ é a covariância genética entre $x$ e $y$, em que, $\hat{\sigma}_{a(x)}^{2}$ é componente de variância genética para o $x$, e $\hat{\sigma}_{a(y)}^{2}$ é a variância genética para o $y$. A correlação fenotípica $\left(\hat{r}_{f}\right)$ entre dois caracteres foi estimada por $\hat{r}_{f}=\frac{C \hat{O} V_{f(x, y)}}{\sqrt{\hat{\sigma}_{f(x)}^{2} \cdot \hat{\sigma}_{f(y)}^{2}}}$ em que $C \hat{O} V_{f(x, y)}$ é a covariância fenotípica entre $x$ e $y, \hat{\sigma}_{f(x)}^{2}$ é o componente de variância fenotípica para o $x$, e $\hat{\sigma}_{f(y)}^{2}$ é o componente de variância fenotípica para o y. As correlações genéticas foram obtidas pelo método REML/BLUP (máxima verossimilhança restrita/melhor predição linear não viesada).

\subsection{Análise dos Modelos Mistos}

Por meio do método de Análise de Deviance avaliou-se a significância estatística entre os tratamentos. Para sua composição foram realizados os seguintes passos (Resende, 2007b): i) obtenção do logaritmo do ponto máximo da função de verossimilhança residual (L) para os modelos com e sem o efeito a ser testado; ii) cálculo da Deviance $(D=-2 \times \log L)$ para modelos com e sem o efeito a ser testado; iii) obtenção da razão de verossimilhança (LR, do inglês Likelihood Ratio) dada pela diferença entre as Deviances para modelos com e sem o efeito a ser testado; iv) teste da significância da diferença entre Deviances via LRT (Likelihood Ratio Test), usando o teste qui-quadrado com um grau de liberdade.

\subsection{Ordenamento dos Clones}

Os valores genotípicos de cada clone foram obtidos somando os efeitos genotípicos à média geral do ensaio. O ganho genético $\left(G_{s}\right)$ equivale à média dos vetores dos efeitos genéticos preditos para os clones selecionados. A média geral $(\mu)$ somada ao $G_{s}$ resultou na média da população melhorada. O desempenho relativo de cada clone foi obtido pela relação entre as médias da população melhorada de cada clone e a média do clone de maior valor genético. Foram acrescentados, na listagem, os valores fenotípicos de cada clone, correspondentes aos valores de campo observados no experimento. A intensidade de seleção clonal utilizada para obtenção da população melhorada foi $10 \%$.

\section{RESULTADOS E DISCUSSÃO}

$\mathrm{O}$ efeito de tratamentos foi significativo a $1 \%$ de probabilidade, indicando a existência de variação genética entre os clones, a qual foi detectada pelo teste da razão de verossimilhança (LRT, $X^{2}=6,63$ ) (Tabela 1). A existência de variação genética é um fator básico aos programas de melhoramento, pois subsidia o progresso genético com a prática da seleção, uma vez que esta variabilidade pode ser explorada para o desenvolvimento de materiais genéticos (Cruz et al., 2004; Rocha et al., 2009). 
TAMBARUSSI, E.V. et al. Seleção de clones de Eucalyptus.

Tabela 1. Análise de Deviance e teste de razão da verossimilhança (LTR) dos parâmetros genéticos de clones de Eucalyptus spp. para os caracteres diâmetro à altura do peito - DAP (cm) e altura - ALT (m) aos quatro (DAP4, ALT4) e aos cinco (DAP5, ALT5) anos de idade.

Table 1. Analysis of Deviance and likelihood ratio test (LTR) of genetic parameters for each variable in relation to diameter at breast height - DAP (cm) and height - ALT (m) of Eucalyptus spp. clones at four (DAP4, ALT4) and five (DAP5, ALT5) years old.

\begin{tabular}{ccccc}
\hline & \multicolumn{4}{c}{ Caractere } \\
\cline { 2 - 5 } Deviance & DAP4 & DAP5 & ALT4 & ALT5 \\
\hline Clone $\left(\mathrm{h}_{2}\right)$ & $4.329,44$ & $5.345,93$ & $3.458,25$ & $3.556,86$ \\
Parcela $\left(\mathrm{c}_{2}\right)$ & $3.544,54$ & $4.215,27$ & $3.044,99$ & $2.867,26$ \\
Modelo & $3.526,67$ & $4.198,8$ & $3.034,38$ & $2.837,74$ \\
LRT $\left(\mathrm{h}_{2}\right)$ & $802,77^{* *}$ & $1147,13^{* *}$ & $423,87^{* *}$ & $719,12^{* *}$ \\
LRT $\left(\mathrm{c}_{2}\right)$ & $17,87^{* *}$ & $16,47^{* *}$ & $10,61^{* *}$ & $29,52^{* *}$ \\
\hline
\end{tabular}

Clone $\left(\mathrm{h}_{2}\right)$ : Deviance do clone; Parcela $\left(\mathrm{c}_{2}\right)$ : Deviance da parcela; Modelo: Deviance do modelo; LRT $\left(\mathrm{h}_{2}\right)$ : Razão da verossimilhança do clone; LRT $\left(\mathrm{c}_{2}\right)$ : Razão da verossimilhança da parcela; **: significativo à $1 \%$ de probabilidade.

Clone $\left(\mathrm{h}_{2}\right)$ : Clone's Deviance; Plot $\left(\mathrm{c}_{2}\right)$ : Plot's Deviance; Model: Model's Deviance; LRT $\left(\mathrm{h}_{2}\right)$ : likelihood ratio of clone; LRT $\left(\mathrm{c}_{2}\right)$ : likelihood ratio of plot; **: significant at $1 \%$ of probability.

Nesse trabalho, o efeito do coeficiente de determinação de parcela $\left(c_{2}\right)$ foi considerado significativo a $1 \%$ de probabilidade para todas as variâncias, indicando a influência do ambiente nas estimativas dos parâmetros genéticos (Tabela 1). Isso certamente ocorreu pelo uso de materiais não adaptados às condições edafoclimáticas da região onde o ensaio foi instalado experimento.

As estimativas das variâncias genotípicas $\left(\hat{V}_{g}\right)$ mostraram que os caracteres avaliados no ambiente em estudo apresentaram taxas de controle genéticos similares (Tabela 2). No caso da variância ambiental entre parcelas $\left(\hat{V}_{c}\right)$ e variância residual $\left(\hat{V}_{e}\right)$, aos quatro e cinco anos de idade, houve pouca variação para ambos os caracteres avaliados (Tabela 2). Esse resultado indica que as variações fenotípicas $\left(\hat{V}_{f}\right)$ encontradas entre os indivíduos são de caráter genético (Garcia e Nogueira, 2005).

O valor da herdabilidade depende de todos os componentes da variância, assim, qualquer alteração em um deles afetará o valor da proporção herdável, que expressa a proporção da variação total que é atribuída ao efeito genético (Falconer, 1989). Os coeficientes de herdabilidade individual no sentido amplo $\left(\hat{h}_{g}^{2}\right)$, ou seja, dos efeitos genotípicos totais encontrados, foram de média magnitude para os caracteres DAP e ALT, variando entre $46 \%$ a $56 \%$ e $40 \%$ a $55 \%$, respectivamente (Tabela 3). Esses resultados são favoráveis à seleção de clones, o que gerará altos ganhos na próxima seleção.

O coeficiente de determinação dos efeitos ambientais entre parcelas $\left(c_{\text {parc }}^{2}\right)$ mensura a variabilidade dentro dos blocos, logo, quanto mais elevado este valor, maior será a variância ambiental (Rocha et al., 2006). Para os caracteres avaliados, aos quatro e cinco anos de idade, os valores foram de baixa magnitude para DAP (de 0,009 a 0,011), enquanto para a altura este parâmetro variou de 0,012 até 0,019, apresentando, portanto, maior variabilidade em relação ao DAP (Tabela 3). 
TAMBARUSSI, E.V. et al. Seleção de clones de Eucalyptus.

Tabela 2. Estimativa das variâncias genéticas (parcela, ambiental e fenotípica) para clones de Eucalyptus spp. para os caracteres diâmetro à altura do peito - DAP $(\mathrm{cm})$ e altura - ALT (m) aos quatro (DAP4, ALT4) e aos cinco (DAP5, ALT5) anos de idade.

Table 2. Estimates of genetic variance (plot, environment and phenotype) of Eucalyptus spp. clones for diameter at breast height - DAP (cm) and height - ALT (m) at four (DAP4, ALT4) and five (DAP5, ALT5) years old.

\begin{tabular}{lllll}
\hline & \multicolumn{3}{c}{ Caractere } \\
\cline { 2 - 5 } Parâmetros & DAP4 & DAP5 & ALT4 & ALT5 \\
\hline$\hat{V}_{g}$ & 1,77 & 3,70 & 1,86 & 2,87 \\
$\hat{V}_{c}$ & 0,04 & 0,06 & 0,06 & 0,10 \\
$\hat{V}_{e}$ & 2,02 & 2,85 & 2,73 & 2,23 \\
$\hat{V}_{f}$ & 3,83 & 6,61 & 4,64 & 5,19 \\
\hline
\end{tabular}

$\hat{V}_{g}$ : variância genotípica; $\hat{V}_{c}$ : variância ambiental entre parcelas; $\hat{V}_{e}$ : variância residual; $\hat{V}_{f}$ : variância fenotípica individual.

$\hat{V}_{g}$ : genotypic variance; $\hat{V}_{c}$ : environmental variance among plots; $\hat{V}_{e}:$ residual variance; $\hat{V}_{f}$ : individual phenotypic variance.

Tabela 3. Estimativa das herdabilidades para clones de Eucalyptus spp. para os caracteres diâmetro à altura do peito - DAP (cm) e altura - ALT (m) aos quatro (DAP4, ALT4) e aos cinco (DAP5, ALT5) anos de idade.

Table 3. Estimates of heritability for Eucalyptus spp. clones for diameter at breast height - DAP (cm) and height - ALT (m) at four (DAP4, ALT4) and five (DAP5, ALT5) years old.

\begin{tabular}{lllll}
\hline & \multicolumn{3}{c}{ Caractere } \\
\cline { 2 - 5 } Parâmetros & DAP4 & DAP5 & ALT4 & ALT5 \\
\hline$\hat{h}_{g}^{2}$ & $0,46 \pm 0,04$ & $0,56 \pm 0,05$ & $0,40 \pm 0,05$ & $0,55 \pm 0,06$ \\
$\hat{h}_{a j}^{2}$ & 0,466 & 0,564 & 0,405 & 0,563 \\
$c_{\text {parc }}^{2}$ & 0,011 & 0,009 & 0,012 & 0,019 \\
$\hat{h}_{m}^{2}$ & 0,994 & 0,996 & 0,993 & 0,995 \\
\hline
\end{tabular}

$\hat{h}_{g}^{2}$ : herdabilidade individual no sentido amplo; $\hat{h}_{a j}^{2}:$ herdabilidade ajustada; $c_{p a r c}^{2}$ : efeitos ambientais entre parcelas; $\hat{h}_{m}^{2}$ : herdabilidade média de clones.

$\hat{h}_{g}^{2}$ : broad-sense heritability; $\hat{h}_{a j}^{2}$ : adjusted heritability; $c_{\text {parc }}^{2}$ : environmental effects amog plots; $\hat{h}_{m}^{2}$ : clonal mean heritability.

A variação genética entre os clones, que é expressa pelo valor do coeficiente de variação genética $\left(C V_{g}(\%)\right)$ em percentuais da média geral, variou de $11,44 \%$ a $14,70 \%$ para o caractere DAP e $9,15 \%$ a $10,22 \%$ para altura, o que pode ser favorável à seleção (Garcia e Nogueira, 2005). Quanto à razão entre $C V_{g} / C V_{e}$, que é expressa pelos valores do coeficiente de variação relativa $(\mathrm{CV})$, os resultados encontrados (Tabela 4) para DAP e altura aos quatro e cinco anos foram superiores a um. Segundo Vencovsky (1987), esse resultado indica que o processo de seleção gerará altos e rápidos ganhos genéticos. 
TAMBARUSSI, E.V. et al. Seleção de clones de Eucalyptus.

Tabela 4. Estimativa dos parâmetros genéticos para clones de Eucalyptus spp. para os caracteres diâmetro à altura do peito - DAP (cm) e altura - ALT (m) e altura (ALT, m) aos quatro (DAP4, ALT4) e aos cinco (DAP5, ALT5) anos de idade.

Table 4. Estimates of genetic parameters for Eucalyptus spp. clones for diameter at breast height $-\mathrm{DBH}(\mathrm{cm})$ and height - H (m) at four (DAP4, ALT4) and five (DAP5, ALT5) years of age.

\begin{tabular}{lllll}
\hline & \multicolumn{3}{c}{ Caractere } \\
\cline { 2 - 5 } Parâmetros & DAP4 & DAP5 & ALT4 & ALT5 \\
\hline$C V_{g}(\%)$ & 11,44 & 14,70 & 9,15 & 10,22 \\
$C V_{e}(\%)$ & 3,82 & 4,02 & 3,46 & 3,12 \\
$C V_{r}$ & 2,99 & 3,65 & 2,63 & 3,27 \\
$A c_{\text {clone }}$ & 0,99 & 0,99 & 0,99 & 0,99 \\
$\hat{r}_{g}$ & 0,99 & 0,99 & 0,99 & 0,99 \\
Média geral & 11,61 & 13,07 & 14,90 & 16,57 \\
\hline
\end{tabular}

$C V_{g}(\%)$ : coeficiente de variação genética; $C V_{e}(\%)$ : coeficiente de variação residual; $C V_{r}$ : coeficiente de variação relativa; $A c_{\text {clone }}$ : acurácia de clone; $\hat{r}_{g}$ : estimativa de correlação genética.

$C V_{g}(\%)$ : coefficient of genetic variation; $C V_{e}(\%)$ : coefficient of residual variance; $C V_{r}$ : coefficient of relative variation; $A c_{\text {clone }}$ : clonal accuracy; $\hat{r}_{g}:$ genetic correlation estimate.

As estimativas de correlações genéticas $\left(\hat{r}_{g}\right)$ entre os caracteres avaliados aos quatro e cinco anos foram altas e positivas; dessa forma um caractere irá beneficiar outros, viabilizando a seleção indireta (Falconer, 1987). Observa-se que o maior valor da média geral dos parâmetros foi para ALT e DAP aos cinco anos. Não houve grande variação no comportamento nas idades avaliadas (Tabela 4). As correlações fenotípicas foram elevadas para os caracteres e anos avaliados (Tabela 5).

$\mathrm{Na}$ seleção, emprega-se o caráter que está menos sujeito a erro, no caso o DAP. Assim, devido à confiabilidade e facilidade na medição, optou-se por conduzir a seleção apenas para o DAP aos três anos de idade, que apresentou alta correlação genética com a altura. Trabalhos com outros clones também priorizaram esse caractere na seleção de materiais genéticos (Pires, 1996; Tambarussi et al., 2017). Para dar continuidade aos testes clonais, os $10 \%$ melhores clones, baseados no DAP aos três anos de idade, foram selecionados e ordenados de acordo com os melhores valores genotípicos (Tabela 6).

Valores genotípicos devem ser utilizados pelos melhoristas, pois são estes os valores verdadeiros associados aos caracteres avaliados e que devem ser, portanto, utilizados para a seleção de materiais mais produtivos. A seleção dos melhores clones pelo método REML/BLUP tem-se mostrando eficiente, resultando em ganhos substanciais no melhoramento florestal (Garcia e Nogueira, 2005; Rocha et al., 2007).

Tabela 5. Matriz de correlação fenotípica para clones de Eucalyptus spp. para os caracteres diâmetro à altura do peito - DAP (cm) e altura - ALT (m), aos quatro (DAP4, ALT4) e cinco (DAP5, ALT5) anos de idade.

Table 5. Phenotypic correlation matrix of Eucalyptus spp. clones for diameter at breast height - DAP (cm) and height - ALT (m), at four (DAP4, ALT4) and five (DAP5, ALT5) years old.

\begin{tabular}{lccc}
\hline Variável & DAP5 & ALT4 & ALT5 \\
\hline DAP4 & $0,96^{*}$ & $0,93^{*}$ & $0,86^{*}$ \\
DAP5 & - & $0,91^{*}$ & $0,88^{*}$ \\
ALT4 & - & - & $0,93^{*}$ \\
\hline
\end{tabular}

*Significativo a 5\% de probabilidade.

*Significative at $5 \%$ of probability. 
TAMBARUSSI, E.V. et al. Seleção de clones de Eucalyptus.

Tabela 6. Ordenamento dos clones selecionados de Eucalyptus spp. com melhor desempenho para diâmetro à altura do peito - DAP $(\mathrm{cm})$ aos três anos de idade.

Table 6. Ranking of Eucalyptus spp. selected clones based on performance of diameter at breast height - DAP (cm) at three years old.

\begin{tabular}{cccccc}
\hline Ordem & Clones & $\mathrm{G}$ & $\mathrm{u}+\mathrm{g}$ & Ganho & Nova Média \\
\hline 1 & 1 & 5.5914 & 18.6657 & 5.5914 & 18.67 \\
2 & 2 & 5.5152 & 18.5895 & 5.5533 & 18.63 \\
3 & $\mathrm{AA}-217$ & 5.0570 & 18.1313 & 5.3878 & 18.46 \\
4 & 1199 & 4.5140 & 17.5882 & 5.1694 & 18.24 \\
5 & 1016 & 4.1661 & 17.2404 & 4.9687 & 18.04 \\
6 & 1004 & 3.6109 & 16.6852 & 4.7424 & 17.81 \\
7 & $\mathrm{~A}$ & 3.5600 & 16.6343 & 4.5735 & 17.65 \\
8 & 1005 & 3.4111 & 16.4854 & 4.4282 & 17.50 \\
9 & 1041 & 2.8830 & 15.9573 & 4.2565 & 17.33 \\
10 & 3 & 2.8350 & 15.9093 & 4.1143 & 17.19 \\
11 & 1044 & 2.5253 & 15.5996 & 3.9699 & 17.04 \\
12 & 1000 & 2.4571 & 15.5313 & 3.8438 & 16.91 \\
13 & $\mathrm{~B}$ & 2.3377 & 15.412 & 3.7280 & 16.80 \\
\hline
\end{tabular}

g: efeito genotípico predito; $\mathrm{u}$ : média dos clones; $\mathrm{u}+\mathrm{g}$ : valor genotípico.

$\mathrm{g}$ : predicted genotypic effect; $\mathrm{u}$ : clone average; $\mathrm{u}+\mathrm{g}$ : genotypic value.

\section{CONCLUSÕES}

Os coeficientes de herdabilidade foram de média magnitude para os caracteres diâmetro à altura do peito - DAP e altura - ALT.

As correlações genéticas e fenotípicas foram altas e positivas, possibilitando tanto a seleção precoce quanto a indireta.

Há boas chances de sucesso na seleção e ganhos genéticos para o material avaliado devido à alta variabilidade genética presente entre os clones.

\section{AGRADECIMENTOS} dos dados.

Ao Grupo Ferroeste pela concessão

\section{REFERÊNCIAS BIBLIOGRÁFICAS}

ANDRADE, M.C. et al. Quantity, organization, and distribution of chloroplast microsatellites in all species of Eucalyptus with available plastome. Crop Breeding and Applied Biotechnology, v. 18, p. 97-102, 2018.
ASSOCIAÇÃO BRASILEIRA DE PRODUTORES DE FLORESTAS PLANTADAS - ABRAF. Anuário estatístico da ABRAF, 2013. Ano base 2012. Brasília, 2012. 147 p.

BERGER, R. et al. Efeito do espaçamento e da adubação no crescimento de um clone de Eucalyptus saligna Smith. Ciência Florestal, v. 12, p. 75-87, 2002.

CRUZ, C.D.; REGAZZI, A.J.; CARNEIRO, P.C.S. Modelos biométricos aplicados ao melhoramento genético. Viçosa, MG: UFV, 2004. v. 1, 480 p.

FALCONER, D.S. Introduction to quantitative genetics. New York: Longman Scientific and Technical, 1989. 438 p.

GARCIA, C.H.; NOGUEIRA, M.C.S. Utilização da metodologia REML/BLUP na seleção de clones de eucalipto. Scientia Forestalis, v. 68, p. 107-112, 2005. 
TAMBARUSSI, E.V. et al. Seleção de clones de Eucalyptus.

INDÚSTRIA BRASILEIRA DE ÁRVORES - IBÁ. Relatório Anual 2016. Brasília, DF: 2016. 100 p. . Relatório IBÁ 2014. São Paulo: IBÁ, 2014.

LIMA, A.A.C.; OLIVEIRA, F.N.S.; AQUINO, A.R.L. Solos e aptidão edafoclimática para a cultura do cajueiro no Município de Grajaú-MA. Fortaleza: Embrapa Agroindústria Tropical, 1999. 5 p.

MARTINI, A.J. Edmundo Navarro de Andrade: o plantador de eucaliptos: a questão da preservação florestal no Brasil. São Paulo: Humanitas FFLCH-USP, 2008. 370 p.

MIRANDA, S.B. A monocultura do eucalipto alterando o espaço agrário no oeste maranhense. In: ENCONTRO NACIONAL DE GEOGRAFIA AGRÁRIA， 21., 2012, Uberlândia. Anais... Disponível em: < http://www.lagea.ig.ufu.br/xxlenga/ anais_enga_2012/eixos/1213_1.pdf $>$ Acesso em: 26 nov 2017.

NUNES, B.H.S. et al. Implicações da interação genótipo $\mathrm{x}$ ambientes na seleção de clones de eucalipto. Cerne, v. 8, p. 49-58, 2002.

PINTO JÚNIOR, J.E. et al. Efeitos da correção de dados na redução da heterogeneidade das variâncias genética, ambiental e fenotípica em testes de progênies de Eucalyptus grandis W. Hill ex Maiden. Pesquisa Florestal Brasileira, n. 51, p. 75-107, 2005.

PIRES, I.E. Eficiência da seleção combinada no melhoramento genético de Eucalyptus spp. 1996. 116 f. Tese (Doutorado em Genética e Melhoramento) - Universidade Federal de Viçosa, Viçosa, MG.

RESENDE, M.D.V.de. SELEGEN-REML/BLUP: sistema estatístico e seleção genética computadorizada via modelos lineares mistos. Colombo: Embrapa Florestas, 2007a. 359 p.

Matemática e estatística na análise de experimentos e no melhoramento genético. Colombo: Embrapa Florestas, 2007b. 362 p.
ROCHA, M.G.B. et al. Avaliação genética de progênies de meio-irmãos de Eucalyptus urophylla utilizando os procedimentos REML/ BLUP e E (QM). Ciência Florestal, v. 16, n. 4, p. 369-379, 2006.

Seleção de genitores de Eucalyptus grandis e de Eucalyptus urophylla para produção de híbridos interespecíficos utilizando REML/BLUP e informação de divergência genética. Revista Árvore, v. 31, n. 6, p. 977-987, 2007.

ROCHA, R.B. et al. Estimação de parâmetros genéticos e seleção de procedências e famílias de Dipteryx alata Vogel (baru) utilizando metodologia de REML-BLUP e E(QM). Cerne, v. 15, n. 3, p. 331-338, 2009.

TAMBARUSSI, E.V. et al. Estimativa de parâmetros genéticos para a seleção precoce em clones de Eucalyptus spp. Scientia Forestalis, v. 45, n. 115, p. 1-10, 2017.

VENCOVSKY, R. Herança quantitativa. In: PATERNIANI, E.; VIEGAS, G.P. (Ed.). Melhoramento e produção de milho. 2. ed. Campinas: Fundação Cargill, 1987. v. 1, p. 137-214.

VERRYN, S.D. Eucalyptus hybrid breeding in South Africa. In: DUNGEY, H.S.; DIETERS, M.J.; NIKLES, D.J. (Ed.). Proceedings of QFRI/ CRC-SPF Symposium: Hybrid Breeding and Genetics of Forest Trees. Noosa: Department of Primary Industries, 2000. p. 191-199.

XAVIER, A.; SILVA, R.L. Evolução da silvicultura clonal de Eucalyptus no Brasil. Agronomía Costarricense, v. 34, p. 93-98, 2010. 